\title{
Chaotic Time Series Prediction Using Immune Optimization Theory
}

\author{
Yuanquan Shi ${ }^{1,2}$, Xiaojie Liu ${ }^{1,}{ }^{*}$, Tao $\mathrm{Li}^{1}$, Xiaoning Peng ${ }^{2}$, Wen Chen ${ }^{1}$, Ruirui Zhang ${ }^{1}$, Yanming Fu ${ }^{1}$ \\ ${ }^{1}$ School of Computer Science, Sichuan University, Chengdu, 610065, China \\ ${ }^{2}$ School of Computer Science, Huaihua University, Huaihua, 418000, China \\ E-mail:syuanquan@163.com,liuxiaojie8@126.com,litao@scu.edu.cn,hhpxn@163.com, \\ cwcwcwcw2006@163.com,ruiruizhang@163.com,fym2005@126.com
}

\begin{abstract}
To solve chaotic time series prediction problem, a novel Prediction approach for chaotic time series based on Immune Optimization Theory (PIOT) is proposed. In PIOT, the concepts and formal definitions of antigen, antibody and affinity being used for time series prediction are given, and the mathematical models of immune optimization operators being used for establishing time series prediction model are exhibited. Chaotic time series is analyzed and corresponding sample space is reconstructed by phase space reconstruction method; then, the prediction model of chaotic time series is constructed by immune optimization theory; finally, using this prediction model to forecast chaotic time series. To demonstrate the effectiveness of PIOT, the three typical chaotic nonlinear time series are generated by nonlinear dynamics systems that are Lorenz, Mackey-Glass and Henon, respectively, and are used for simulating prediction. The simulation results show that PIOT is a feasible and effective prediction method, and meanwhile provides a novel prediction approach for chaotic time series.
\end{abstract}

Keywords: Chaotic time series, phase space reconstruction, immune optimization theory, prediction.

\section{Introduction}

Time series is a set of data constructed by chronological observed data. Generally speaking, time series prediction mainly includes three operation steps, namely analyzing the past and current data of time series, establishing the corresponding time series prediction model, and predicting the future data of time series. Time series prediction has received considerable applications in various fields ${ }^{1,2,3}$, such as economics prediction, weather prediction and network traffic prediction etc. Time series prediction may usually be classified into the linear and nonlinear prediction method. The typical linear time series prediction models mainly include auto regressive (AR), moving average (MA), auto regressive moving average (ARMA) and so forth. ${ }^{3}, 4,5$ However, these models are mostly the statistical method based prediction models and need some priori knowledge that are proved difficultly, so these models can hardly obtain effective prediction results for chaotic nonlinear time series. Due to the most of time series possessing chaotic nonlinear characteristics in real-life and artificial intelligence method holding better approximation capability for nonlinear time series, numerous researchers have proposed using artificial intelligence method to solve nonlinear time series prediction problem, such as genetic algorithm $(\mathrm{GA})^{6}$, neural network $(\mathrm{NN})^{7}$, support

* Corresponding author.

E-mail: liuxiaojie8@126.com (Xiaojie Liu). 
vector machine $(\mathrm{SVM})^{8}$ and so on. The advantage of GA is that it can establish prediction model by means of a small amount of data of time series, but its shortcomings show that it exists some limiting problems, for instance, the simple evolutionary learning mechanism. The merits of NN have mainly self-learning, nonlinear mapping and parallel distributed processing etc. but it has some congenital drawbacks, such as easy to fall into local optimization, difficult to determine the number of hidden layers and slow to training speed. ${ }^{2,9}$ Although SVM has some merits which are strong predicting ability, fast convergence rate and global optimization etc, but the prediction performance of SVM is sensitive to some key parameters that are penalty parameter, non-sensitive loss parameter and kernel function and so on. ${ }^{10,11,12}$

Artificial immune system (AIS) is another new frontier research in artificial intelligence areas, and is one of bionic intelligent systems inspired by biological immune system (BIS). It can not only distinguish and withstand nonself antigens that are illegal intrusions but also possess the evolutionary learning mechanism of Darwin's evolution theory ${ }^{13,14}$. The study of AIS mainly includes negative selection, clonal selection theory and artificial immune network ${ }^{15}$. To date AIS has been successfully employed in a wide variety of different application fields like network security, numerical optimization, data mining, pattern recognition, fault detection and so forth. ${ }^{16,17,18,19,20}$ AIS make full use of some merits of BIS such as self-learning, self-adaption, self-organization and immune memory etc. In order to resist nonself antigen and maintain immune system stability, antibody will be cloned, mutated or produced continually so as to enhance the restraint ability against nonself antigens. ${ }^{14}$ According to the advantages of AIS, many researchers have proposed intelligence methods based on immune optimization mechanism for solving corresponding nonlinear problems, where the specific nonlinear problem is viewed as antigen and the candidate solution of relevant problems is viewed as antibody. ${ }^{21,}{ }^{22}$ Compared with other intelligence methods, AIS possesses the capabilities of the global optimization and fast convergence speed. ${ }^{23,24}$

In this paper, a novel prediction approach for chaotic time series based on immune optimization theory (PIOT) is proposed according to the chaotic characteristics of nonlinear time series and the superiority of artificial immune system solving nonlinear problems. The mainly contributions of this paper are: 1) Propose a novel prediction modelling method for chaotic time series based on phase space reconstruction method and immune optimization theory; 2) Introduce a new antibody encoding form corresponding with time series prediction problem that inspired by the antibody characteristics of BIS; 3) Give a new immune selection operator in terms of artificial immune network, the new operator introduces affinity not only between antibody and antigen but between antibody and antibody in order to enhance the diversity of population; 4) Give a new antibody mutation operator according to the new antibody encoding form, the new operator utilizes affinity between antibody and antigen to determine antibody mutation strategy so as to improve the capabilities of the global search and local optimization for population.

The remainder of this paper is organized as follows. The prediction principle of chaotic time series is described in Section 2. The prediction approach for chaotic time series based on immune optimization theory (PIOT) is presented in Section 3. The effectiveness of PIOT is verified using three typical chaotic time series generated by nonlinear dynamic systems in Section 4. Finally, the conclusion is given in the last section.

\section{The Prediction Principle of Chaotic Time Series}

Chaotic time series prediction process is mainly composed of time series analysis, prediction modelling and time series prediction. The change for any data element of chaotic time series is decided by interacting with neighbored data elements. So the inherent variation rule of time series can be found by analyzing the subsequence of time series before prediction point, and a prediction value that approximate target value at prediction point will be deduced by it. Packard et al. in Ref. 25 have put forward the concept of phase space reconstruction and introduced the chaotic theory to analyze nonlinear time series, the idea of phase space reconstruction is that the phase space of time series is reconstructed using the delay coordinate of one of variables in dynamic system observed. Then, Takens et al. in Ref. 26 have given the relevant mathematical proof for phase space reconstruction theory, the conclusions show that the regular track may being restored from dynamic system by an appropriate 
embedding dimension to reconstruct a high-dimensional phase space. Therefore, phase space reconstruction theory provides a reliable theory foundation for chaotic time series prediction problems.

Utilizing phase space reconstruction method to analyze and reconstruct chaotic time series, a suitable phase space (i.e. sample space) being used for establishing time series prediction model is obtained. Suppose chaotic time series $X=\{x(t) \mid x(t) \in \mathbb{R}\}$, where $t=1,2 \ldots \mathrm{n} ; \mathbb{R}$ is the real number set, a sample space $S$ established by phase space reconstruction method is:

$$
\{X(t), Y(t)\}=\{[x(t-\tau), x(t-2 \tau), \cdots, x(t-d \tau)],[x(t)]\} .
$$

Where $t=n, n-\tau, \ldots,(d+1) \tau ; \tau$ is the time delay; $d$ is the embedding dimension; $X(t)$ is the in-sample of prediction model; $Y(t)$ is the target value of prediction model; sample space $S$ is composed of $n-(d-1) \tau$ samples.

For chaotic time series prediction, the prediction value is more affected by neighboring data elements than far distance data elements for time series, so the performance of establishing prediction model and corresponding prediction precision can be improved by selecting appropriate training samples (i.e. sample window) neighbored with prediction point. In this paper, we adopt C-C method ${ }^{27}$ to calculate time delay, embedding dimension and delay time window (i.e. sample window). Let $w(\leq l)$ is the sample window size, where $l=n-(d-1) \tau$ represents the sample number of sample space $S$, when consider corresponding prediction value to target value $Y(t), w$ samples before prediction point $t$ are selected to build time series prediction model $f: R^{d} \rightarrow R$, and then forecasting target value at $t$ time, the relationship between prediction value and prediction model is:

$$
\hat{Y}(t)=f(x(t-\tau), x(t-2 \tau), \cdots, x(t-d \tau)) .
$$

\section{Immune Optimization based Chaotic Time Series Prediction}

As the above-mentioned prediction principle, the vital problem of chaotic time series prediction is how to build an efficient prediction model by the past and current data of time series. To solve chaotic time series prediction problem, a novel prediction approach for chaotic time series based on immune optimization theory (PIOT) is proposed. The basic ideas of PIOT are: 1) to analyze chaotic nonlinear time series and reconstruct a high-dimensional sample space by phase space reconstruction method introduced in Section 2;2) to approximate the sample point of sample space by immune optimization mechanism such as clonal selection, immune network, immune memory and vaccination, and then build corresponding prediction model of time series; 3 ) to predict time series using this prediction model. Therefore, the prediction modelling process of time series realized by immune optimization mechanism is a crucial part of PIOT.

\subsection{PIOT modelling}

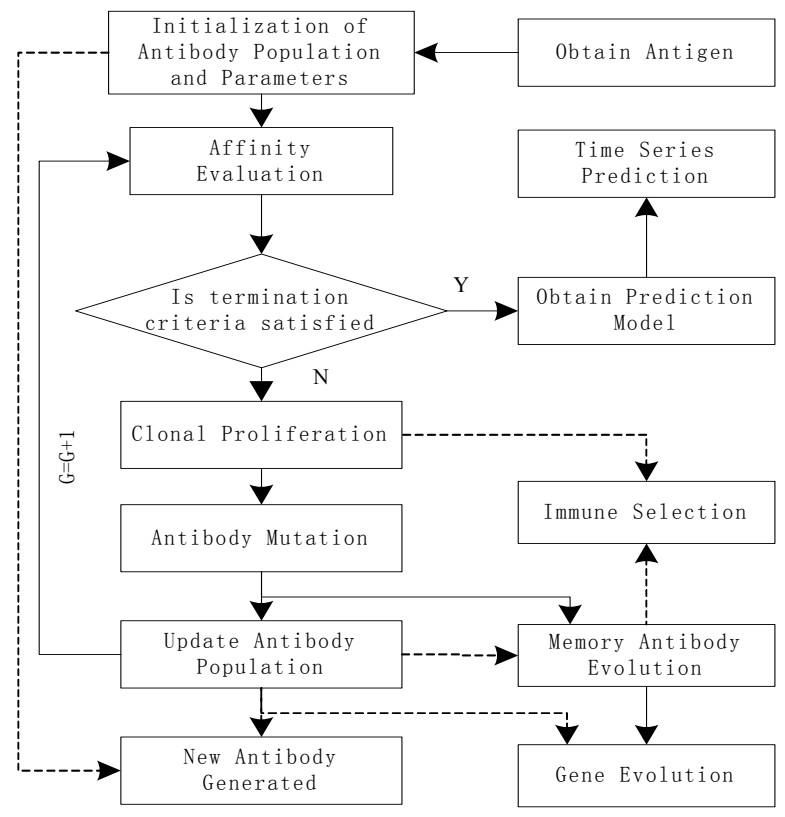

Fig. 1. The prediction modelling paradigm of PIOT.

The prediction modelling paradigm of PIOT is shown in Fig.1. The function modules of PIOT mainly include initialization of antibody population and parameters, affinity evaluation, immune selection, clonal proliferation, antibody mutation, memory antibody and gene evolution etc. In Fig.1, the solid lines indicate the basic flowchart of prediction modelling; the dotted lines indicate the called relationship between both function modules, namely one function module linking with the arrow tail need to call another function module linking with the arrow head in modelling process. When affinity between antibody and antigen is reach to the termination condition of immune evolution, the prediction modelling process is stopped and the relevant prediction model is obtained. Thus, the modelling capability and 
the prediction performance of PIOT depend on antibody encoding mechanism, affinity evaluation method and immune optimization operators etc.

In this section, the concepts and formal definitions of antigen, antibody and affinity associated with time series is given. Then, the mathematical models of immune optimization operators being used for time series prediction modelling, which deal with immune selection, clonal proliferation, antibody mutation, memory antibody evolution and gene evolution, are depicted in detail.

\subsubsection{Antigen, antibody and affinity}

In BIS, antigen is a kind of substances which can stimulate organism to produce immune response and arouse specific reaction associated with the organism's reactant; and this substance can be recognized as exogenous substances or some components of organism. Antigen holds two important characteristics ${ }^{28}$ : 1) immunogenicity, i.e. antigen stimulates lymphocyte of immune system to produce immune response; 2) specific reactivity, i.e. antigen is specifically synthesized with antibody. Antibody is a type of immune molecule which is produced by humoral immune response after antigen stimulating organism, namely immunoglobulin. Antibody can not only directly neutralize antigen components such as pathogens, microbe and virus, but also induce cellular immune response. Due to the specificity of antigen, there are antigenic determinants on antigen surface which can be recognized by antibodies, and meanwhile antibody determinants on antibody surface being used for recognizing antigenic determinants. Thus, affinity is usually defined as discrimination intensity between antigenic determinant and antibody determinant.

In order to simulate the dynamic immune mechanism of BIS, this paper applies immune optimizing mechanism to solve chaotic time series prediction problem. The concepts and formal definitions of antigen, antibody and affinity being used for establishing time series prediction model are depicted in this section.

\subsubsection{Antigen}

The definition of antigen is relative to the specific problem in AIS. For chaotic time series prediction problem, this paper use Takens' delay embedding theory to analyze time series $X=\{x(t) \mid x(t) \in \mathbb{R}, t \in \mathbb{N}\}$ and reconstruct corresponding sample space $S$. In the course of prediction, a part of samples in sample space $S$ is viewed as antigen, which is the premise condition of time series prediction modelling.

Definition 1. Antigen atg $=\left\{a g_{i} \mid a g_{i} \in \mathbb{R}^{d+1}, i \in m\right\}$ represents a training sample subset in sample space $S$ being used for establishing time series prediction model; antigen component $a g=<x_{1}, x_{2}, \cdots, x_{d}, x_{d+1}>$ represents a sample point in training sample subset.

Where $d$ is the embedding dimension, $m$ is the number of antigen component. An antigen component comprises the in-sample $X(t)$ and target value $Y(t)$ associated with prediction model.

\subsubsection{Antibody encoding}

Antibody is made up of the Fab(Fragment antigenbinding) and the $F c$ (Fragment crystallized) in BIS. ${ }^{28}$ The $F a b$ of antibody, which can higher bind with antigen, is formed by two identical polypeptide heavy chains and two identical polypeptide light chains. Each polypeptide comprises the constant region and the variable region, where the constant region is relatively stable parts for the number and arrangement of amino acids on polypeptide chain; the variable region is a part of amino acids that its arrangement is different for different antibody. Furthermore, the variable region is divided into the hypervariable region and the framework region, the studies of biological immunology show that antibody mutation reaction is only concentrated in hypervariable region including a few number of amino acids, and the amino acid variation of framework region is relatively less than that of hypervariable region. Accordingly the antibody fragment of hypervariable region has better affinity with antigen, and is a crucial region of antibody in order to recognize antigens. The biological structure of antibody can abstractly be depicted in Fig. 2.

\begin{tabular}{|l|l|l|}
\hline \multicolumn{2}{|c|}{ Variable Region } & \\
\hline Hypervariable Region & Framework Region & Constant Region \\
\hline
\end{tabular}

Fig. 2. The biological structure of antibody.

For chaotic time series prediction problem, the prediction model is usually represented as mathematical expression, its elements is mainly composed of arithmetic operators, mathematical functions, variables, constants and so forth, so the precision of prediction model is related to the structure and the constituted elements of mathematical expression. Besides the linear 
expression, the expression tree is another representing form of mathematical expression. ${ }^{29}$ The expression tree has two type nodes: the leaf nodes and the non-leaf nodes, the mapping relationship between the linear expression and the expression tree that its maximum out-degree equals two is shown in Fig. 3. According to Fig. 3(b), the symbol change of non-leaf node has a vital effect to the operation result and the structure of mathematical expression. For time series prediction model, once prediction model changed, viz., the variation rule of time series was destroyed, the corresponding prediction result may be deviated seriously. On the contrary, the symbol change of leaf node can bring only slight effect to the operation result of mathematical expression, and not change to the structure of that.

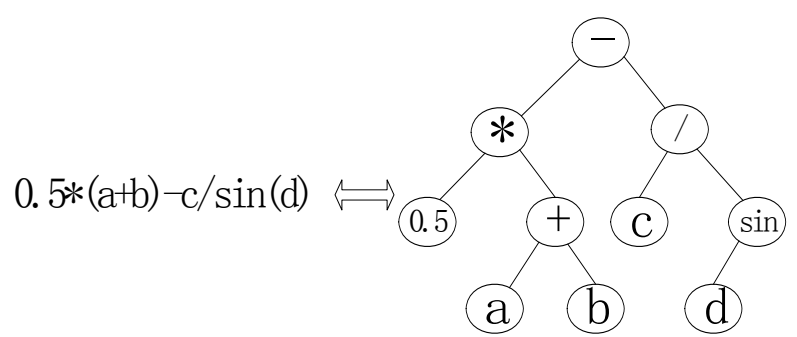

(a) linear expression

(b) expression tree

Fig. 3. Mapping relationship of linear expression and expression tree.

According to the biological structure of antibody and the mapping relationship between the expression tree and the linear expression, the formal definition of antibody encoding used for chaotic time series prediction problem is depicted detailedly as follows.

Definition 2. Antibody $a t b=\{\langle\mathrm{Hr}, \mathrm{Fr}, \mathrm{Cr}\rangle \mid \mathrm{Hr} \in$ $\left.\{\text { Funs, Vars }\}^{L_{h}}, F r \in\{\text { Vars }\}^{L_{f}}, C r \in \mathbb{N}^{L_{c}}\right\} \quad$ denotes a candidate solution of prediction model being used for approximating time series curve, viz., a character string containing prediction model; Population $A b s=\left\{a t b_{i} \mid 1 \leqslant i \leqslant \mathrm{n}\right\}$.

Where $\mathrm{Hr}, \mathrm{Fr}$ and $\mathrm{Cr}$ denote respectively the hypervariable region, the framework region and the constant region of antibody; Funs $\subseteq\{+,-, *, /, Q, P, N, O\}$ denotes a set which include arithmetic operators and mathematical functions selected by user in terms of a given problem complexity, $Q$ represents the square root function, $P$ represents the exponential function, $N$ represents the sine function and $O$ represents the cosine function; Vars $\subseteq\{?, a, b, c, \cdots, x, y, z\}$ denotes the variable set specified by user according to a given problem parameters, ? represents the coefficient location in prediction model; $\mathbb{N}$ denotes natural number set; $L_{h}, L_{f}$ and $L_{c}$ (constant natural number) denote respectively the length of hypervariable region, framework region and constant region for an antibody; $n$ (constant natural number) denotes the number of population.

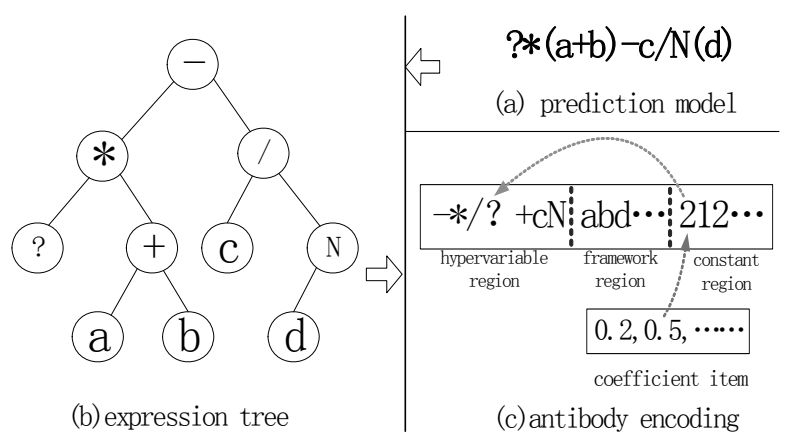

Fig. 4. Mapping relationship of prediction model, expression tree and antibody encoding.

From definition 2, the structure of antibody encoding is composed of hypervariable region, framework region and constant region. For the symbols of antibody encoding, the hypervariable region involves arithmetic operators, mathematical functions, variables and ?; the framework region involves variables and ?; the constant region only involves constant natural number. For example, a prediction model, $0.5^{*}(\mathrm{a}+\mathrm{b})-\mathrm{c} / \sin (\mathrm{d})$, can be converted into ?* $(\mathrm{a}+\mathrm{b})-\mathrm{c} / \mathrm{N}(\mathrm{d})$, the mapping relationship of prediction model, expression tree and antibody encoding are shown in Fig. 4, where Fig. 4(a) presents prediction model; Fig. 4(b) is a expression tree associated with Fig. 4(a) that its maximum out-degree equals two; according to the structure characteristics of antibody and the hierarchical traverse method of tree ${ }^{29}$, a antibody encoding consisting of some possessing specific meaning characters in Fig. 4(c) is obtained by Fig. 4(b). Known from Fig. 4, the antibody decoding operation is a reverse process of antibody encoding.

The key factor to determine the length of antibody encoding is variable region according to the definition and characteristics of antibody encoding. Due to the prediction model being one part of antibody encoding, the length of prediction model will not exceed the predefined length of antibody encoding. This paper uses some characteristics of expression tree to determine the length of variable region that is the node number of expression tree. The expression tree comprise non-leaf 
node (function node) and leaf node (terminal node), non-leaf node is mainly related to arithmetic operator and mathematical function etc, and leaf node usually includes variable,? and so on, where ? represents the coefficient of prediction model.

Theorem 1. Supposing $F_{n c}$ represents the node number of expression tree; $m$ represents the maximum argument number of function node, then the maximum number of terminal node $T_{n c}$ do not exceed $(m-1) * F_{n c}+1$.

Proof. Let an expression tree holds $n$ nodes, the maximum out-degrees of node equal $m, n_{i}$ indicates the node number that the out-degrees of node equal $i$, so the node number of expression tree is

$$
n=n_{0}+n_{1}+n_{2}+\cdots+n_{m} .
$$

In expression tree, except for root node, the all rest nodes have an entry filiation. Let $s$ indicates the sum of filiation, then $n=s+1$. Due to these filiations are related to nodes that their out-degrees respectively are $1,2,3, \ldots$, $m$, viz., the sum of filiation $s=n_{1}+2 n_{2}+3 n_{3}+\ldots+m n_{m}$, the node number of expression tree is

$$
n=n_{1}+2 n_{2}+3 n_{3}++m n_{m}+1 \text {. }
$$

From Eqs. (3) and (4), the number of terminal node that their out-degrees equal zero is

$$
n_{0}=n_{2}+2 n_{3}+3 n_{4}+\cdots+(m-1) n_{m}+1 .
$$

Hence, when the out-degree of each function node in expression tree equal $m$, in other words, $n_{2}=n_{3}=\ldots=n_{m-1}=0$, we can obtain

$$
n_{0}=(m-1) n_{m}+1 \text {. }
$$

Where $n_{m}$ represents the function node number; $n_{0}$ represents terminal node number.

Therefore, the maximum number of terminal node $T_{n c}$ do not exceed $(m-1) * F_{n c}+1$.

From theorem 1, the variable region length is the sum of function nodes and terminal nodes for expression tree in the worst case, i.e. $F_{n c} * m+1$, where function node number $F_{n c}$ is given by user. So hypervariable region length $L_{h}$ and framework region length $L_{f}$ in this paper equal respectively function node number $\left(F_{n c}\right)$ and terminal node number $\left(T_{n c}\right)$. According to the hierarchical traverse method of expression tree, we consider two situations about the encoding symbols of hypervariable region: 1) when the function node number is less than the hypervariable region length, the encoding symbols comprise arithmetic operators, mathematical functions and variables; 2) when the function node number equal the hypervariable region length, the encoding symbols include only arithmetic operator and mathematical function. Moreover, the encoding symbol form of framework region corresponds with the terminal node symbol of expression tree.

The constant region is mainly related to two concepts: index item index and coefficient item coefficient, its length can be given by user according to the complexity of problem. The constant region $C r=\left\{\forall\right.$ index $_{i} \mid$ index $_{i} \in$ Scefindex, $\left.1 \leqslant i \leqslant L_{c}\right\}$ is an index item set comprising index values, the coefficient item set Scoefficient $=\left\{\right.$ coefficient $_{j} \mid$ coefficient $_{j} \in \mathbb{R}$, $j \in$ Scefindex $\}$ is a real number set, where Scefindex $\subseteq \mathbb{N}$ is the range of subscript value of coefficient item, $\mathbb{N}$ is natural number set, $\mathbb{R}$ is real number set, $L_{c}\left(\leq L_{h}\right)$ is the constant region length. Each index value corresponds to each coefficient location in coefficient item set, and is used for obtaining real value of corresponding coefficient item in prediction model, the variation rang of coefficient value is between $[0,1]$ The relationship of index item, coefficient item and antibody encoding symbol '?' is shown in Fig. 4.

\subsubsection{Affinity evaluation}

Affinity is usually used for describing the fitness or similarity between antigen and antibody in AIS. As far as chaotic time series prediction problem is concerned, using affinity between antibody and antigen to reflect the approximate intensity between prediction models and time series, and using affinity both antibodies to reflect the population diversity.

Definition 3. Antigen-affinity is defined as the fitness between antibody and antigen; antigen-affinity is evaluated by

$$
A f f_{a t g}(a t b, a t g)=\frac{1}{1+\sqrt{\frac{1}{m} \sum_{j=1}^{m}\left(p_{j}\left(a t b, a g_{j}\right)-t_{j}\right)^{2}}} .
$$

Where $a g_{j}$ is the $j$ th component of antigen $a t g$, $1 \leq \mathrm{j} \leq m ; p_{j}$ is the prediction value obtained by using the in-sample $X(t)$ associated with prediction model in antigen component $a g_{j}$ to replace corresponding variables of prediction model included in antibody $a t b$; $t_{j}$ is the target value $Y(t)$ associated with prediction model in antigen component $a g_{j} ; \sqrt{(\bullet)}$ is the root mean square error(RMSE) between target values and 
prediction values. The smaller $\sqrt{(\bullet)}$, the bigger antigenaffinity, it show antibody has better fitness to antigen, viz., prediction model holding better approximation capability to time series. The antigen-affinity is close to 1 when $\sqrt{(\cdot)}$ is gradually decreased to zero, i.e. the prediction model associated with a given antibody is an idea prediction model.

From definition 1 and definition 2, an in-sample $X(t)$ in antigen component exists corresponding relationship with a variable character of antibody encoding. To calculate antigen-affinity, each antigen component ag effectively combines with the given antibody according to Eq. (7). Taking the approximation capability between antigen component and antibody as example, supposing the embedding dimension $d=4$, then an in-sample $X(t)$ of antigen component can be expressed as $\langle x(1), x(2), x(3), x(4)\rangle$, the variable character set of antibody encoding is defined as $\{a, b, c$, $\mathrm{d}\}$, where $\mathrm{a}, \mathrm{b}, \mathrm{c}$ and $\mathrm{d}$ correspond to $x(1), x(2), x(3)$ and $x(4)$, respectively. It is worth noting: data number of insample part of antigen component equal variable number corresponding with antibody encoding, and keeps consisting with the embedding dimension $d$. Let $x(1)=0.22, \quad x(2)=0.63, \quad x(3)=0.12, \quad x(4)=0.85, \quad$ the prediction model, ?* $(\mathrm{a}+\mathrm{b})-\mathrm{c} / N(\mathrm{~d})$, in Fig. 4 can be rewritten as $?^{*}(0.22+0.63)-0.12 / \sin (0.85)$, where ? equals 0.5 , viz., obtaining a prediction value associated with antigen component $a g$.

Definition 4. Antibody-affinity is defined as the similarity between one antibody and another antibody in population; antibody-affinity is evaluated by

$$
\begin{aligned}
& A f f_{a t b}\left(a t b_{s}, a t b_{t}\right)=\frac{\sum_{i=1}^{l e n_{a b}} f_{\text {match }}\left(a t b_{s}(i), a t b_{t}(i)\right)}{\operatorname{len}_{a t b}} . \\
& f_{\text {match }}\left(a t b_{s}(i), a t b_{t}(i)\right)=\left\{\begin{array}{l}
0, \text { iff } \text { atb }_{s}(i) \neq a t b_{t}(i) \\
1, \quad \text { otherwise }
\end{array}\right.
\end{aligned}
$$

Where len $_{a t b}=L_{h}+L_{f}+L_{c}, L_{h}, L_{f}$ and $L_{c}$ represent hypervariable region length, framework region length and constant region length of an antibody, respectively. Generally, antibody-affinity is calculated by hamming distance method.

\subsubsection{Immune optimization operators}

According to clonal selection theory, the classical clonal selection algorithm mainly relates to simple immune operators such as selection, clone, mutation and so on. ${ }^{18}$
In the process of the prediction modelling of chaotic time series based on immune optimization theory, this paper proposed corresponding immune optimization operators used for solving time series prediction problem, which are immune selection, clonal proliferation, antibody mutation, memory antibody evolution and gene evolution. Using these operators to realize immune evolution operation, they can not only improve the global search capability but also expedite the convergence speed of antibody evolution.

\subsubsection{Immune selection}

To solving the optimization problem of some application engineering areas using clonal selection idea, the classical clonal selection algorithm usually use affinity between antigen and antibody to change the population diversity so as to find the global optimization solution of a specific problem. The classical clonal selection algorithm mainly adopts two immune optimization operations, namely hypermutation and receptor editing, to improve the population diversity in order to enhance the recognizing capability of antibody for antigen. Due to immune selection operation being the precondition of immune evolution, the suitable immune selection operation has a guiding effect for immune evolution. For evolution selection mechanism, the traditional selection methods mainly include roulette algorithm, elite selection, bipolar selection, level-based selection and so on. ${ }^{14}$ To realize the clonal proliferation operation, de Castro et al. proposed to select $n$ high antigen-affinity antibodies from population ${ }^{18}$; Nitesh Khilwani et al. introduced a power based roulette selection algorithm to select antibodies from population $^{24}$; Gong et al. proposed to select high antigen-affinity antibodies proportionally from population ${ }^{21}$.

Inspired by immune network theory ${ }^{14,15,19}$, a new immune selection operator is proposed. Due to this operator introducing antigen-affinity and antibodyaffinity at the same time, this operator can provide chance to select not only high antigen-affinity antibodies but also low antigen-affinity antibodies in order to realize the diversity of population; and simultaneously can avoid the phenomenon of repeated operations for the similar antibody to some extent. From definition 4, population is divided into multiple subpopulations according to the similarity of antibody, and the stimulated level of antibody in population is 
calculated using the concentration of similar antibodies in sub-population. Therefore, the probability of antibody selected is related to the stimulated level of antibody and the antigen-affinity.

Supposing $A b s$ indicates population; $a t b$ indicates antibody; $n$ indicates the population size; $\alpha$ indicates the threshold of similar antibody, then the stimulated level of antibody $a t b_{s}(\in A b s)$ is calculated by Eqs. (10) and (11).

$$
\begin{aligned}
& A b s_{s u b}=\left\{a t b \mid A f f_{a t b}\left(a t b_{s}, a t b\right) \geq \alpha\right. \\
& \left.\wedge a t b_{s} \in A b s \wedge \forall a t b \in A b s-a t b_{s}\right\} \\
& \operatorname{Sti}\left(a t b_{s}\right)=\frac{\left|A b s_{s u b}\right|}{n} . \\
& P_{\text {select }}\left(a t b_{s}\right)=\frac{A f f_{\text {atg }}\left(a t b_{s}, a t g\right)}{\sum_{i=1}^{n} A f f_{\text {atg }}\left(a t b_{i}, a t g\right)} \cdot e^{-S t i\left(a t b_{s}\right)} .
\end{aligned}
$$

Where Eq. (10) is used for searching a subpopulation $A b s_{\text {sub }}$ from population $A b s$, and the antibody-affinity between each antibody of $A b s_{\text {sub }}$ and antibody $a t b_{s}$ is not less than $\alpha$; Eq. (11) is used for calculating the stimulated value $S t i$ of $a t b_{s}$ in $A b s_{s u b}$. From Eq. (12), the selection expectation value $P_{\text {select }}$ for $a t b_{s}$ which is selected to the next step evolution operation is directly proportional to the antigen-affinity and inversely proportional to the stimulated value $S t i$, where $\left|A b s_{\text {sub }}\right|$ represents antibody number of $A b s_{\text {sub }}$. In population, an antibody holding higher the antigenaffinity and lower the stimulated value Sti has more chance being selected to the next step evolution operation, reversely, it will be restrained. And meanwhile lower antigen-affinity antibodies have chance being selected to the next step evolution operation too. The immune selection algorithm (ISA) is depicted in Algorithm 1.

Algorithm 1. The immune selection algorithm (ISA).

\begin{tabular}{ll}
\hline Step 1: & $\begin{array}{l}\text { Use Eq. }(8) \text { to calculate the antigen-affinity of } \\
\text { population } A b s ;\end{array}$ \\
Step 2: & Calculate the stimulated value $S t i$ of population $A b s ;$ \\
Step 2.1: & $\begin{array}{l}\text { Use Eq. }(10) \text { to obtain a sub-population } A b s_{s u b} \text { from } \\
\text { population } A b s, \text { and each antibody of } A b s_{s u b} \text { hold } \\
\text { same antibody-affinity with antibody } a t b_{s} ;\end{array}$ \\
Step 2.2: & $\begin{array}{l}\text { Use Eq. }(11) \text { to calculate the stimulated value } S t i \text { of } \\
\text { antibody } a t b_{s} \text { in population } A b s ;\end{array}$
\end{tabular}

Step 2.3: $\quad$ If existing an antibody that its stimulated value Sti has not being calculated in population $A b s$, go to Step 2.1 ;

Step 3: $\quad$ Use Eq. (12) to calculate the selection expectation value $P_{\text {select }}$ of population $A b s$ being selected to the next step evolution operation.

\subsubsection{Clonal proliferation}

In clonal proliferation operation, antibody being sorted as descending order according to its selection expectation value $P_{\text {select }}$, and then selecting $m$ antibodies from current generation population, which hold higher $P_{\text {select }}$, to be used for clonal proliferation in terms of the clone ratio $R_{c}\left(0<R_{c}<1\right)$, viz., constituting a selection antibody population $A b s_{\text {select }}$ that include $m$ antibodies. Known from ISA, the clonal proliferation operation can ensure that not only the higher antigen-affinity antibodies in population are cloned but also the lower antigen-affinity antibodies have chance to be cloned too, so that enlarging the searching space and improving the searching speed for population. Eqs. (13) and (14) represent respectively antibody clonal number $N_{\text {clone }}$ and clonal antibody population $\mathrm{Abs}_{\text {clone }}$.

$$
\begin{gathered}
N_{\text {clone }}=\sum_{i=1}^{m}\lceil(n-i) / i\rceil . \\
A b s_{\text {clone }}=\left\{e_{i} \times a t b_{i} \mid\right. \\
\left.a t b_{i} \in A b s_{\text {select }}, i=1,2, \cdots, m\right\}
\end{gathered}
$$

Where $n$ is the antibody population size; $i$ is the index value of the selected antibody in $A b s_{\text {select }}$, the smaller the number $i$, the bigger the antigen-affinity and the more the clonal number of antibody; $N_{\text {clone }}$ is the sum of clonal antibody for $m$ higher antigen-affinity antibodies; $e_{i}$ is a $\lceil(n-i) / i\rceil$-dimensional identify column vector; $A b s_{\text {clone }}$ is the clonal antibody population generated by $m$ selected antibodies.

\subsubsection{Antibody mutation}

Antibody mutation operation is the most critical part of immune evolution. Antibody mutation can not only maintain the diversity of population but also guide the global optimization of population in immune evolution. Mutation operation can occur in any position within antibody encoding. The optimization antibody solution associated with the given problem can be quickly found by effective mutation operation.

During immune evolution, to avoid convergence early or local optimization for population, we need 
consider the diversity of population from the whole of antibody encoding; reversely, to search the superior antibody from local optimization space to suffice the specific problem, we need consider the micro-variability of antibody from the part-specified of antibody encoding. ${ }^{14,} 30$ According to the characteristics of antibody encoding in definition 2, we adopt two mutation strategies: local mutation and global mutation. The local mutation only considers to mutate the hypervariable region of lower antigen-affinity antibody, and to improve the antigen-affinity of antibody by the global searching of antibody space. For the higher antigen-affinity antibody, the global mutation can improve the antigen-affinity of antibody by local searching of antibody space so as to finding the optimization antibody. The global mutation only considers mutating slightly the framework region and constant region of antibody, the mutation operation does not cause seriously deviation to antigen-affinity of antibody.

For antibody mutation process, using Eq. (15) to select the mutation strategy in terms of the antigenaffinity of antibody in $A b s_{\text {clone, }}$, where $\beta$ in Eq. (16) represents the average antigen-affinity of $m$ selected antibodies in $A b s_{\text {select }}$. If antigen-affinity is less than $\beta$, $M t=1$ indicates the local mutation of antibody; reversely, the mutation strategy of antibody is regarded as the global mutation. The difference between global mutation and local mutation is exhibited by the different mutation regions within antibody encoding. If the mutation operation happens on hypervariable region of antibody relating to the local mutation, the structure of prediction model within antibody will be changed and meanwhile the antigen-affinity of antibody will be mutated too. Moreover, to the framework region and constant region relating to the global mutation, the aim of mutation operation makes the antigen-affinity of antibody obtaining a little rising. To realize the mutation operation of antibody, using the mutation probability defined by Eq. (17) to mutate a gene within antibody encoding, so that enhancing the population diversity and improving the antigen-affinity of antibody. The mutated antibodies constitute a mutation antibody population $A b s_{\text {mutation, }}$ and the antibody mutation algorithm (AMA) is depicted in Algorithm 2.

$$
M t\left(a t b_{i}\right)=\left\{\begin{array}{rr}
1 & A f f_{a t g}\left(a t b_{i}\right)<\beta \\
0 & \text { otherwise }
\end{array} .\right.
$$

$$
\begin{gathered}
\beta=m^{-1} \cdot \sum_{i=1}^{m} A f f_{\text {atg }}\left(a t b_{i}\right) . \\
P_{m}(a t b)=e^{-\operatorname{Affinity}(a t b)} .
\end{gathered}
$$

Theorem 2. For antibody mutation operation, the probabilit $P\left(A b s_{\text {clone }} \rightarrow A b s_{\text {mutation }}\right)$ that the clonal antibody population Abs clone is translated into the mutation antibody population $A b s_{\text {mutation }}$ is greater than 0.

Proof. Supposing $A b s_{\text {clone }}=\left\{a_{i} \mid i \in m\right\}, A b s_{\text {mutation }}=\left\{b_{i} \mid i \in\right.$ $m\}$, known from Eqs. (7) and (17), $P_{m}\left(a_{i}\right) \equiv$ $P_{m}\left(\operatorname{Aff} f_{\text {atg }}\left(a_{i}, a t g\right)\right)>0$. From definition 2, let the length of binary encoding both $a_{i}$ and $b_{i}$ equals $l$, the hamming distance between $a_{i}$ and $b_{i}$ is $d$, known from Ref. 31, the mutation probability that is from $a_{i}$ to $b_{i}$ is: $P\left\{a_{i} \rightarrow b_{i}\right\} \equiv$ $P_{m}\left(a_{i}\right)^{d}\left(1-P_{m}\right)^{l-d}>0$. Hence, there must being a constant $\zeta(0<\zeta<1)$ can suffice:

$$
P\left(A b s_{\text {clone }} \rightarrow A b s_{\text {mutation }}\right)=\prod_{i=1}^{m} P\left\{a_{i} \rightarrow b_{i}\right\} \geq \zeta .
$$

Therefore, the transformation probability from $A b s_{\text {clone }}$ to $A b s_{\text {mutation }}$ is greater than 0 .

Algorithm 2. The antibody mutation algorithm (AMA).

\begin{tabular}{ll}
\hline Step 1: & $\begin{array}{l}\text { Obtain a clonal antibody population } A b s_{\text {clone }} \text { being } \\
\text { used for antibody mutation operation; }\end{array}$ \\
Step 2: & Use Eqs. (15) and (16) to determine antibody \\
& mutation strategy; \\
Step 3: & Mutation operation for $A b s_{\text {clone; }}$ \\
Step 3.2: & $\begin{array}{l}\text { If antigen-affinity of antibody is less than } \beta \text {, antibody } \\
\text { is mutated by local mutation method, viz., the gene } \\
\text { bit within hypervariable region of antibody is mutated } \\
\text { by Eq. (17); }\end{array}$ \\
& $\begin{array}{l}\text { If antigen-affinity of antibody is not less than } \beta, \\
\text { antibody is mutated by global mutation method, viz., } \\
\text { the gene bit within the framework region and constant } \\
\text { region of antibody is mutated by Eq. (17); }\end{array}$ \\
& $\begin{array}{l}\text { Get a mutation antibody population } A b s_{\text {mutation }} \text { by } \\
\text { Step 4: }\end{array}$ \\
&
\end{tabular}

\subsubsection{Memory antibody evolution}

Compared with the genetic evolution mechanism, the significant merits of immune memory operation are that the superior antibodies of population will be stored. By the local optimization to superior antibody, the convergence capability of population is improved. The memory antibody can obtain higher antigen-affinity after clonal proliferation and antibody mutation for current generation population. Memory antibody warehouse $A b s_{\text {memory }}$ is used for storing some selected optimization antibodies from each generation evolution 
population. With the evolution of antibody continually, the antigen-affinity of antibodies in $A b s_{\text {memory }}$ increase gradually, so the optimization antibody according with the specific problem is found finally. Consider antigenaffinity of antibody in population may be degenerated after immune evolution operations such as clonal proliferation, antibody mutation, firstly incorporating the selection antibody population $A b s_{\text {select }}$ to the mutation antibody population $A b s_{\text {mutation, }}$ viz., $A b s_{\text {mutation }}=A b s_{\text {mutation }} \cup\left\{a t b \mid a t b \in A b s_{\text {select }} \wedge a t b\right.$ $\left.\notin A b s_{\text {mutation }}\right\}$, and then calculating renewedly antigen-affinity of $A b s_{\text {mutation }}$ and sorting its antibodies as descending order. A part of antibodies selected from $A b s_{\text {mutation }}$ constitute an update antibody population $A b s_{\text {update }}$ being used for updating $A b s_{\text {memory }}$ in terms of the memory antibody selection ratio $R_{m}$. By memory antibody evolution operation, the higher antigen-affinity antibody in $A b s_{\text {update }}$ will be substituted for the lower antigen affinity antibody in $A b s_{\text {memory. }}$. The basic model of memory antibody evolution is described as follows:

$A b s_{\text {memory }}(t)=A b s_{\text {new }}(t) \cup$

$\left\{\begin{array}{l}A b s_{\text {memory }}(t-1), \quad \text { iff } m l \geq\left(m n+\left|A b s_{\text {new }}(t)\right|\right) \\ \left\{a t b_{i}\left|a t b_{i} \in A b s_{\text {memory }}(t-1), i=1,2, \cdots, m l-\right| A b s_{\text {new }}(t) \mid\right\}, \text { otherwise }\end{array}\right.$

$$
\begin{aligned}
A b s_{\text {new }}(t)= & \left\{a t b \mid a t b \in A b s_{\text {update }}(t) \wedge\right. \\
& \left.A f f_{\text {atg }}(a t b)>A f f_{\text {atg }}(x) \wedge \exists x \in A b s_{\text {memory }}(t-1)\right\}
\end{aligned}
$$

For Eqs. (19) and (20), $t$ is the number of evolution generation, the memory antibody warehouse $A b s_{\text {memory }}(t)$ is set to $\varnothing$ at time $t=0$. In current generation, $A b s_{\text {new }}(t)$ is a memory antibody update population, and its antibodies are a part of higher antigen-affinity antibodies of $A b s_{\text {update }}(t)$ that are not less than the lowest antigen-affinity antibody of $A b s_{\text {memory }}(t-1)$. In the course of each generation evolution for population, we should consider two evolution methods for $A b s_{\text {memory }}(t)$ : If $m l>m n+\left|A b s_{\text {new }}(t)\right|, A b s_{\text {memory }}(t)$ is a union of $A b s_{\text {memory }}(t-$ 1) and $A b s_{\text {new }}(t)$; reversely, substituting $\left|A b s_{\text {new }}(t)\right|$ antibodies of $A b s_{\text {new }}(t)$ for the lower antigen-affinity antibodies of $A b s_{\text {memory }}(t-1)$, i.e. obtaining $A b s_{\text {memory }}(t)$. When an optimization antibody to adapt the specific antigen has no being found, a part of antibodies selected from $A b s_{\text {memory }}(t)$ will be inherited to next generation population so that the optimization antibody can quickly be sought by memory antibody evolution operation.

\subsubsection{Gene evolution}

Definition 5. Gene gen $=<$, cht $>$ represents a segment within antibody encoding, where $g \subset\{F u n s, \operatorname{Vars}, \mathbb{R}\}$ represents an excellent gene molecular, cnt $\in \mathbb{N}$ represents the using frequency of $g$ being used for vaccination during immune evolution.

The reason that gene being introduced into population is gene within antibody encoding possessing some genetic merits. The superior genes can rapidly spread in population and accelerate the convergence speed of population. Gene evolution mainly relate to the gene extraction, the gene warehouse update and the gene vaccination. The gene extraction objectives consider only the superior antibodies which have been updated to memory antibody warehouse, and the extracted gene is a part of excellent encoding segment coming from the hypervariable region of higher antigenaffinity antibody. On the other hand, for gene vaccination operation, during update next generation population, we select a part of excellent genes from gene warehouse being used for gene vaccination for a part of new antibodies so that the antigen-affinity of antibody is improved. It is noteworthy that gene vaccination operation happens only on the hypervariable region and the framework region of antibody. The function of gene evolution is that excellent gene is extracted continually from superior antibodies and the gene warehouse is updated dynamically so as to realizing gene warehouse evolution. The basic model of gene evolution is shown as following:

$$
G n d(t)=\left\{\begin{array}{lr}
\varnothing, & t=0 \\
\text { Tempd }-G n d_{\text {dead }}(t) \cup G n d_{\text {new }}(t), & t \geqslant 1
\end{array} .\right.
$$

Tempd $=$ Tempd $\cup\{$ gen $\mid$ gen $\in$ Gnd $(t-1)$,

$$
\text { gen.cnt }=\left\{\begin{array}{c}
\text { gen.cnt }+ \text { p,iff gen.g } \subset a t b_{i} \wedge a t b_{i} \in \bigcup_{i=1}^{p} a t b_{i} \\
\wedge a t b_{i} \in A b s(t) \\
\text { gen.cnt }-1, \quad \text { otherwise }
\end{array}\right\} .
$$

$$
\begin{aligned}
& \text { Gnd }_{\text {new }}(t)= \\
& \quad \text { gen } \mid \text { gen } \notin \text { Gnd }(t-1), \\
& \text { gen.cnt }=\max \{\text { atb } \in \text { gen.cnt } \wedge \text { gen } \in \text { Tempd }\}\} . \\
& \text { Gnd }_{\text {dead }}(t)=\{\text { gen } \mid \min \{\text { gen.cnt } \wedge \text { gen } \in \text { Tempd }\}\} .
\end{aligned}
$$


From Eqs. (21), (22), (23) and (24), the initial gene warehouse $\operatorname{Gnd}(t)$ is $\varnothing$ at time $t=0$; Tempd is the temporary gene warehouse and is used for accumulating the using frequency cht for genes of previous generation gene warehouse which have been used by some antibodies of current generation population $A b s(t)$. If a gene in $\operatorname{Gnd}(t-1)$ is found in $p$ antibodies of $A b s(t)$, cnt associated with a specific gene add $p$, reversely, cnt subtract 1; $\operatorname{Gnd}_{\text {new }}(t)$ is used for storage superior genes of antibody extracted from $A b s_{\text {new }}(t)$, and its gene using frequency is set to the highest gene using frequency of Gnd $(t-1)$ so that the added new gene will not be early deleted from gene warehouse; $G n d_{\text {dead }}(t)$ is used for storage the lower using frequency genes deleted from $\operatorname{Gnd}(t-1)$. Finally, we ought to notice the uniqueness of gene in Gnd.

\subsection{PIOT algorithm}

Immune optimization theory based chaotic time series prediction algorithm is depicted in Algorithm 3, this algorithm includes four parts: original time series data normalization, time series phase space reconstruction, prediction modelling and time series prediction.

Algorithm 3. The algorithm of PIOT.

\begin{tabular}{|c|c|}
\hline Step 1: & Obtain and normalize original chaotic time series; \\
\hline Step 2: & $\begin{array}{l}\text { Analyze time series using phase space reconstruction } \\
\text { method, and then get reconstructed time series sample } \\
\text { space; }\end{array}$ \\
\hline Step 2.1: & $\begin{array}{l}\text { Obtain corresponding parameters for time series } \\
\text { prediction by C-C method: time delay, embedding } \\
\text { dimension and sample window; }\end{array}$ \\
\hline Step 2.2: & $\begin{array}{l}\text { Reconstruct a reasonable time series sample space by } \\
\text { Eq. (1); }\end{array}$ \\
\hline Step 3: & Establish prediction model for chaotic time series; \\
\hline Step 3.1: & $\begin{array}{l}\text { Get multiple samples before prediction sample point, } \\
\text { the sample number equal the sample window size; }\end{array}$ \\
\hline Step 3.2: & $\begin{array}{l}\text { Establish chaotic time series prediction model sufficing } \\
\text { the user-specified problem by immune optimization } \\
\text { theory based prediction modelling method; }\end{array}$ \\
\hline Step 4: & Predict chaotic time series. \\
\hline
\end{tabular}

From Algorithm 3, the key parts of this algorithm mainly include chaotic time series analysis based on phase space reconstruction and the time series prediction modelling based on immune optimization theory. For time series analysis, we use C-C method obtaining phase space reconstruction parameters, namely time delay and embedding dimension, and then reconstructing a high dimension phase space of time series in order to obtain an efficient time series prediction model. In the course of the prediction modelling of time series, we establish an appropriate prediction model using corresponding immune optimization operators such as clonal proliferation, antibody mutation, memory antibody evolution and gene evolution etc. In this paper, we adopt respectively single-step prediction and multi-step prediction to demonstrate the validity of Algorithm 3, where the single-step prediction method is defined as predicting only one data of the future time sequence according to the past and current time sequence data; the multi-step prediction method is defined as predicting multiple data of the future time sequence, and the prediction values of all former prediction points will be viewed as history time sequence data when the corresponding prediction values of latter prediction points are calculated.

\section{Simulation and Results}

\subsection{Data preprocessing and evaluation criterion}

In the process of simulation experiments, this paper use Eq. (25) to normalize original chaotic nonlinear time series, where $x_{\max }$ and $x_{\min }$ indicate respectively the maximum value and the minimum value of time series, the variation range of normalized time series values is between $[0,1]$.

$$
\bar{x}(t)=\frac{x(t)-x_{\min }}{x_{\max }-x_{\min }} .
$$

To demonstrate the accuracy of PIOT, we introduce three prediction evaluation criterions: the absolute error $(A E)$, the root mean square error (RMSE) and the mean absolute error $(M A E)$. The corresponding definitions are respectively Eqs. (26), (27) and (28).

$$
\begin{gathered}
A E=|\hat{x}(t)-x(t)| . \\
M A E=\frac{1}{n} \sum_{t=1}^{n}|\hat{x}(t)-x(t)| . \\
R M S E=\sqrt{\frac{1}{n} \sum_{t=1}^{n}(\hat{x}(t)-x(t))^{2}} .
\end{gathered}
$$




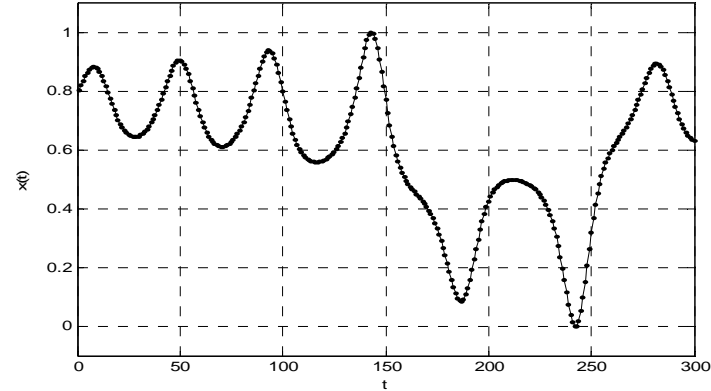

Fig. 5. Lorenz time series.

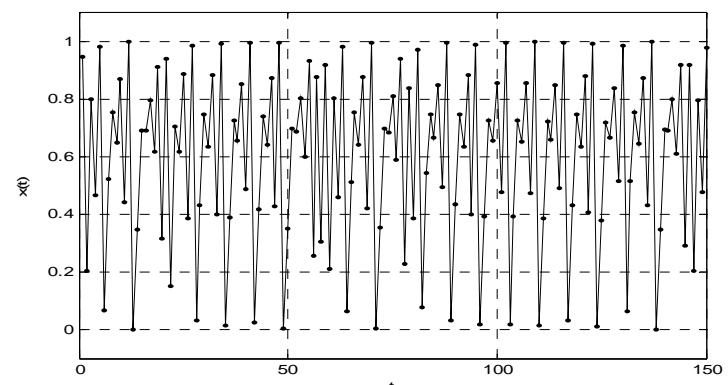

Fig. 7. Henon time series.
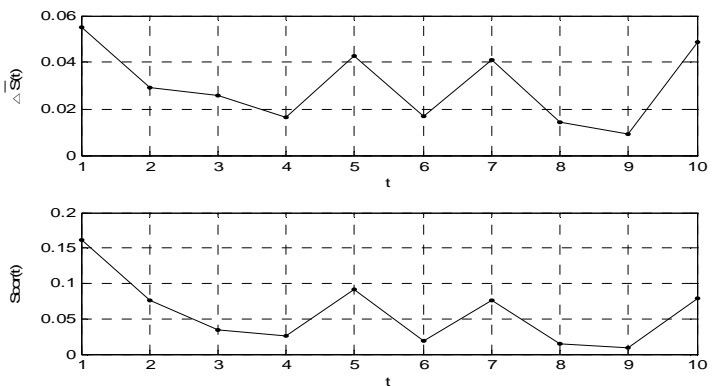

Fig. 9. C-C method analyzing Mackey-Glass time series.

Where $n$ is the number of time series prediction point, $x(t)$ and $\hat{x}(t)$ are the target value and the prediction value of the $t$ th prediction point respectively. $A E$ reflects directly the deviation of target value and prediction value; $M A E$ reflects the average value of prediction errors; and RMSE is used for evaluating the predicting performance of different prediction methods for same prediction objective.

\subsection{Data sets used}

In this paper, we use three typical chaotic time series as benchmark problems, which are Lorenz time series ${ }^{27}$,

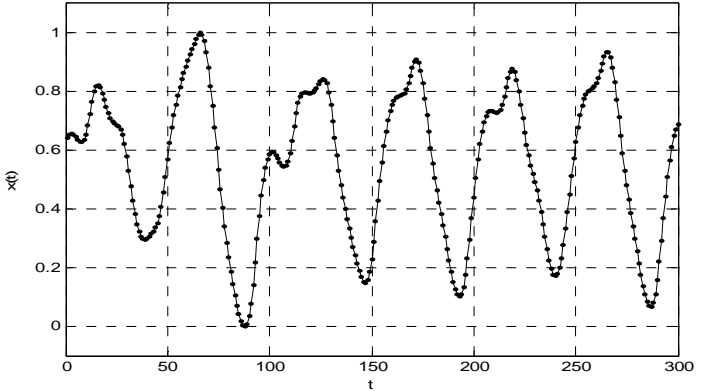

Fig. 6. Mackey-Glass time series.
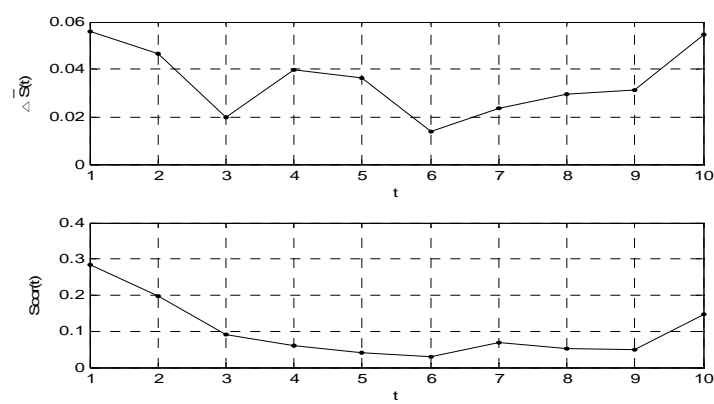

Fig. 8. C-C method analyzing $\mathrm{x}$ component of Lorenz time series.
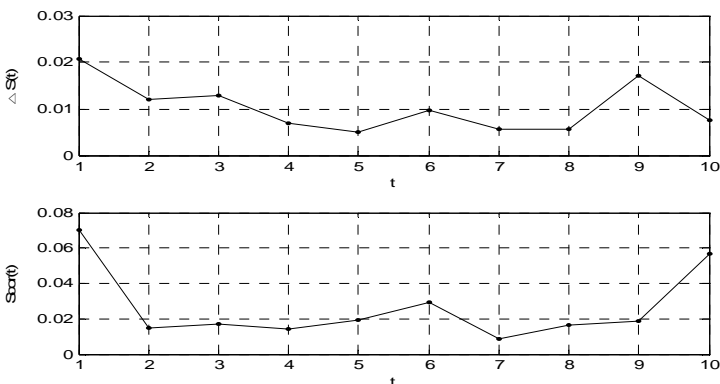

Fig. 10. C-C method analyzing $\mathrm{x}$ component of Henon time series.

Mackey-Glass time series ${ }^{8}$ and Henon time series ${ }^{12}$, to validate the effectiveness of PIOT.

Lorenz time series

\subsubsection{Lorenz time series}

Lorenz system is a deterministic system holding chaotic phenomenon that is found in 1963, the Lorenz system can be described as follows:

$$
\left\{\begin{array}{l}
\dot{x}(t)=a(y(t)-x(t)) \\
\dot{y}(t)=b x(t)-y(t)-x(t) z(t) . \\
\dot{z}(t)=x(t) y(t)-c z(t)
\end{array}\right.
$$


Where $x$ represents the amplitude of convection motion and is directly proportional to the motion intensity; $y$ represents the temperature between the ascending and descending currents; $z$ represents the distortion of the vertical temperature profile from linearity. When $a, b$ and $c$ equal respectively $16,45.92$ and 4 , the system possess chaotic features. Supposing initial condition $x(0)=-1, y(0)=0, z(0)=1$, the integral time step equal $0.01 \mathrm{~s}$, we use the standard fourth-order Runge-Kutta method to solve Eq. (29) and generate chaotic time series by the $x$ component of Eq. (29). For Lorenz time series prediction, we select 8300 data according to Eq. (29), where the first 8000 data are discarded; the rest 300 data are considered as experiment data and its normalized results is shown in Fig. 5.

\subsubsection{Mackey-Glass time series}

Mackey-Glass equation is a delay dynamical system holding chaotic phenomenon that is discovered in 1977 by Mackey and Glass, the Mackey-Glass equation can be described as follows:

$$
\frac{d x(t)}{d t}=\frac{a x(t-\tau)}{1+x^{c}(t-\tau)}-b x(t) .
$$

Where $\tau$ represents the delay parameter, Eq. (30) possess chaotic behavior when $\tau>17$, and $\tau$ is directly proportional to the chaotic intensity of Eq. (30). The parameters, $a, b, c$ and $\tau$, are set to $0.2,0.1,10$ and 17, respectively. For Mackey-Glass time series prediction, we select 8000 data generated by Eq. (30), where the first 7700 data are discarded; the rest 300 data are considered as experiment data, and its normalized results is shown in Fig. 6.

\subsubsection{Henon time series}

Henon mapping is one of the most famous simple dynamical systems. Henon mapping equation can be described as follows:

$$
\left\{\begin{array}{l}
x(t+1)=1-A x^{2}(t)+y(t) \\
y(t+1)=B x(t)
\end{array} .\right.
$$

When the parameters, $A$ and $B$, are respectively fixed at 1.29 and 0.3 , Henon mapping equation shows chaotic behavior. Let initial condition $\mathrm{x}(0)=0$ and $y(0)=0$, we take the $x$ component of Eq. (31) as chaotic time series. For Henon time series prediction, we select
4450 data generated by Eq. (31), where the first 4300 data are discarded; the rest 150 data are considered as experiment data, and its normalized results is shown in Fig. 7.

\subsection{Experiment parameter settings}

In the process of chaotic time series prediction modelling based on immune optimization theory, the simulation experiment mainly relate to two class parameters: the phase space reconstruction parameters and the antibody evolution parameters. The corresponding parameters associated with chaotic time series prediction are analyzed and set as following.

\subsubsection{Parameters of phase space reconstruction}

For Lorenz time series, Mackey-Glass time series and Henon time series, we use $\mathrm{C}-\mathrm{C}$ method to obtain corresponding phase space reconstruction parameters: time delay $\tau$ and embedding dimension $d$. By calculating the statistics average deviation $\Delta \bar{S}(t)$ and the desired value $S_{c o r}(t)$ using C-C method, the variations of $\Delta \bar{S}(t)$ and $S_{c o r}(t)$ for the three time series are shown in Fig. 8, Fig. 9 and Fig. 10 respectively, where the time $t$ corresponding with the first local minimum value of $\Delta \bar{S}(t)$ is regarded as time delay $\tau$; the time $t$ corresponding with the global minimum value of $S_{c o r}(t)$ is regarded as sample window $w$. Therefore, known from the embedding dimension $d=\lceil w / \tau\rceil+1$, the phase space reconstruction parameters of the three time series are given in Table 1.

Table 1. The phase space reconstruction parameters.

\begin{tabular}{llll}
\hline Time series & Time delay $\tau$ & $\begin{array}{l}\text { Embedding } \\
\text { dimension } \boldsymbol{d}\end{array}$ & $\begin{array}{l}\text { Sample } \\
\text { window } \boldsymbol{w}\end{array}$ \\
\hline Lorenz & 3 & 3 & 6 \\
Mackey-Glass & 4 & 4 & 9 \\
Henon & 2 & 5 & 7 \\
\hline
\end{tabular}

\subsubsection{The parameters of antibody evolution}

For chaotic time series prediction modelling, the required immune evolution parameters of PIOT are listed in Table 2 . These parameter settings reasonably will affect the search capability of the optimal antibody. The evolution process of antigen-affinity and the sensitivity analysis of antibody evolution parameters are described in the following section, respectively. To avoid bias, the prediction results of chaotic time series equal to the average forecasting results of $T$ independent trials. 
Table 2. The antibody evolution parameters.

\begin{tabular}{ll}
\hline Parameter & Value \\
\hline Number of runs $T$ & 50 \\
Number of generations $\eta$ & 500 \\
Population size $N$ & 60 \\
Antibody hypervariable region length $L_{h}$ & 25 \\
Antibody framework region length $L_{f}$ & 26 \\
Antibody constant region length $L_{c}$ & 9 \\
Antigen-affinity threshold value $\gamma$ & 0.98 \\
Antibody similarity threshold value $\alpha$ & 0.80 \\
Clonal rate $R_{c}$ & 0.1 \\
Memory antibody selection rate $R_{m}$ & 0.3 \\
\hline
\end{tabular}

\subsubsection{Evolution of affinity}

We select the $x$ component of Lorenz time series to analyze the evolution of antigen-affinity of PIOT. According to the value of antibody evolution parameters listed in Table 2, Fig. 11 gave the evolution process of antigen-affinity in order to obtain an optimal antibody which relates to the prediction model of time series. When the number of generations $\eta=44$, the antigenaffinity is 0.9858 , and reach to the threshold value of antigen-affinity $\gamma$, that is, an optimal antibody is found; When the number of generations $\eta \geqslant 137$, the maximum antigen-affinity is 0.9948 . The experiment results reveals PIOT holds better approximation capability for chaotic time series.

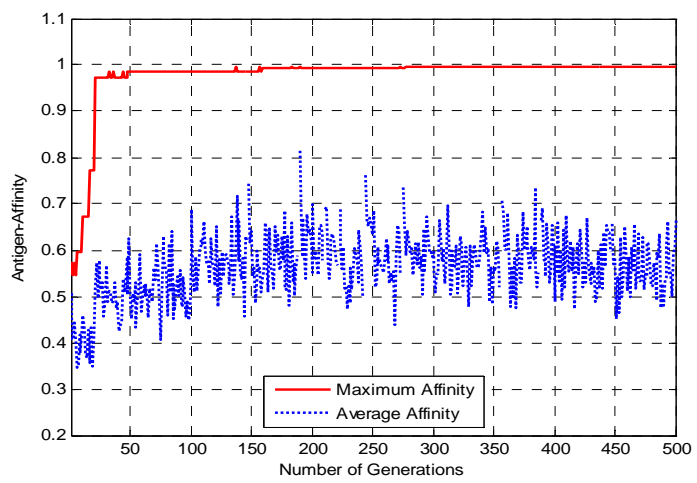

Fig. 11. Evolution of antigen-affinity in PIOT algorithm.

\subsubsection{Sensitivity analysis}

To examine the sensitivity of antibody evolution parameters of PIOT, we select the $\mathrm{x}$ component of Lorenz time series to analyze the sensitivity of the corresponding parameters. Known from Table 2, the parameters that produce biggish impact for the convergence rate of PIOT mainly include population size, antibody hypervariable region length, clonal rate and memory antibody selection rate. So, we consider studying the sensitivity of these parameters below.

\subsubsection{Effect of population size}

In order to examine the effect of population size $N$ for the convergence rate of PIOT, its value is varied from 20 to 500 with an increment of 10 . Fig. 12 show the number of generations required to produce an optimal solution which relates to the prediction model of time series is plotted against the population size. The iteration times used for searching an optimal solution will decrease gradually when the population size increase, that is to say, the convergence rate of PIOT is improved effectively. But when the population size is larger than 50, the iteration times is not exceed to 30 in the worst case and the convergence rate of PIOT is relatively stable.

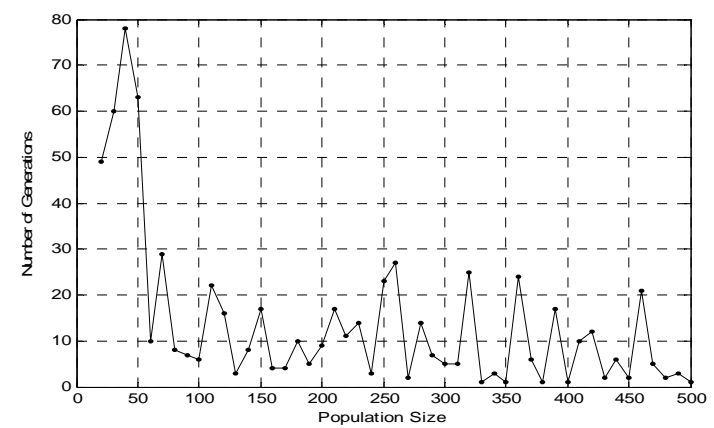

Fig. 12. Sensitivity of Population Size $N$.

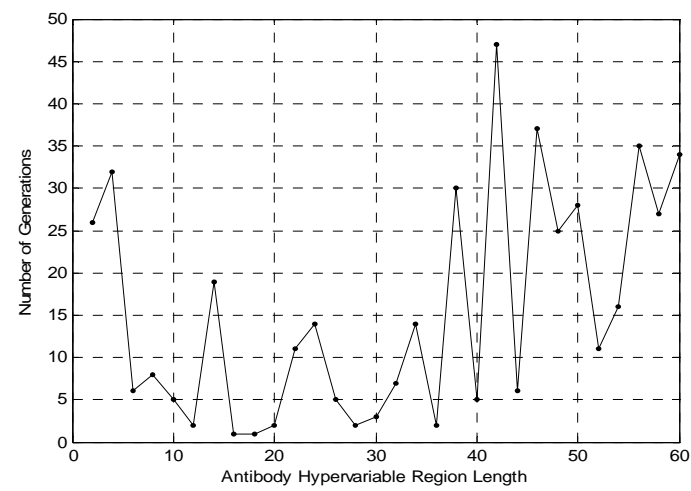

Fig. 13. Sensitivity of Antibody Hypervariable Region Length $L_{h}$.

\subsubsection{Effect of antibody hypervariable region length}

To demonstrate the effect of antibody hypervariable region length $L_{h}$ for the convergence rate of PIOT, its value is varied from 2 to 60 with an increments of 2 . Fig. 
Table 3. The prediction error comparison of chaotic time series.

\begin{tabular}{llllllllllll}
\hline \multicolumn{2}{l}{ Time series } & \multicolumn{3}{l}{ Lorenz } & \multicolumn{7}{c}{ Mackey-Glass } \\
\hline \multicolumn{2}{l}{ Prediction step } & 1 & 20 & 50 & 1 & 20 & 50 & 1 & 20 & 50 \\
\hline \multirow{2}{*}{ RMSE } & PIOT & 0.0081 & 0.0205 & 0.0297 & 0.0084 & 0.0173 & 0.0227 & 0.0562 & 0.0698 & 0.1052 \\
& PGA & 0.0192 & 0.0221 & 0.0309 & 0.0165 & 0.0631 & 0.0406 & 0.1605 & 0.2078 & 0.2487 \\
\multirow{2}{*}{ MAE } & PIOT & 0.0053 & 0.0160 & 0.0256 & 0.0069 & 0.0157 & 0.0167 & 0.0400 & 0.0517 & 0.0725 \\
\multicolumn{2}{l}{} & PGA & 0.0123 & 0.0192 & 0.0273 & 0.0135 & 0.0269 & 0.0348 & 0.1162 & 0.1485 & 0.2183 \\
\hline
\end{tabular}

13 show the number of generations required to produce an optimal solution which relates to the prediction model of time series is plotted against the antibody hypervariable region length. Known from Fig. 13, the variation curve of the number of generations was of a "U" shape, and when the value of antibody hypervariable region length is varied from 6 to 36 , the PIOT hold better convergence rate.

\subsubsection{Effect of antibody clonal rate}

For the clonal rate $R_{c}$ of population, its value is varied from 0.1 to 0.99 with an increment of 0.01 . Fig. 14 show the number of generations required to produce an optimal solution which relates to the prediction model of time series is plotted against the clonal rate. Known from Fig. 14, the number of generations was inapparent increased according to the variation of clonal rate, but we can see the variation curve of the number of generations was overall upward tendency. The experiment results show that the convergence rate of PIOT would be reduced because of the ascending of the clonal rate.

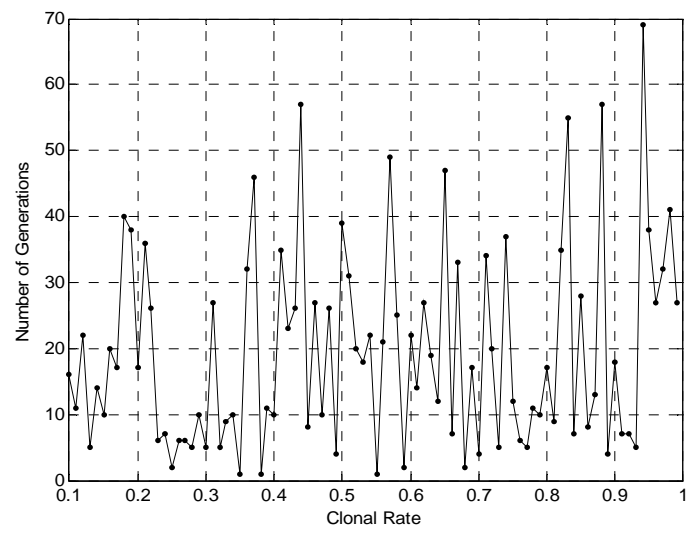

Fig. 14. Sensitivity of Clonal Rate $R_{c}$.

\subsubsection{Effect of memory antibody selection rate}

This session examines the effect of memory antibody selection rate $R_{m}$ for the convergence rate of PIOT; and its value is varied from 0.1 to 0.5 with an increment of 0.01 . Fig. 15 show the number of generations required to produce an optimal solution which relates to the prediction model of time series is plotted against the memory antibody selection rate. Known from Fig. 15, with the growth of memory antibody selection rate, the variation curve of the number of generations was overall downward tendency. The experiment results show that the convergence rate of PIOT would be enhanced due to the ascending of the memory antibody selection rate.

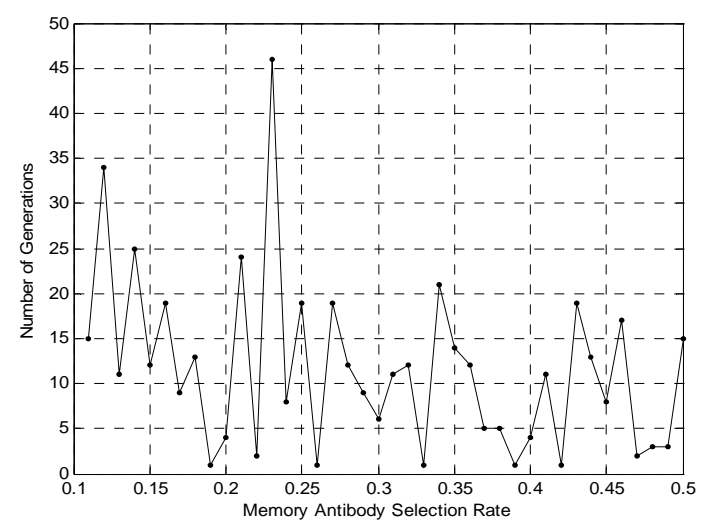

Fig. 15. Sensitivity of Memory Antibody Selection Rate $R_{m}$.

\subsection{Results and analysis}

The three chaotic time series, namely Lorenz time series, Mackey-Glass time series and Henon time series, are considered as experiment test objectives, their variation curves are shown respectively in Fig. 5, Fig. 6 and Fig. 7. Using the corresponding values of the delay time and the embedding dimension listed in Table 1 to 

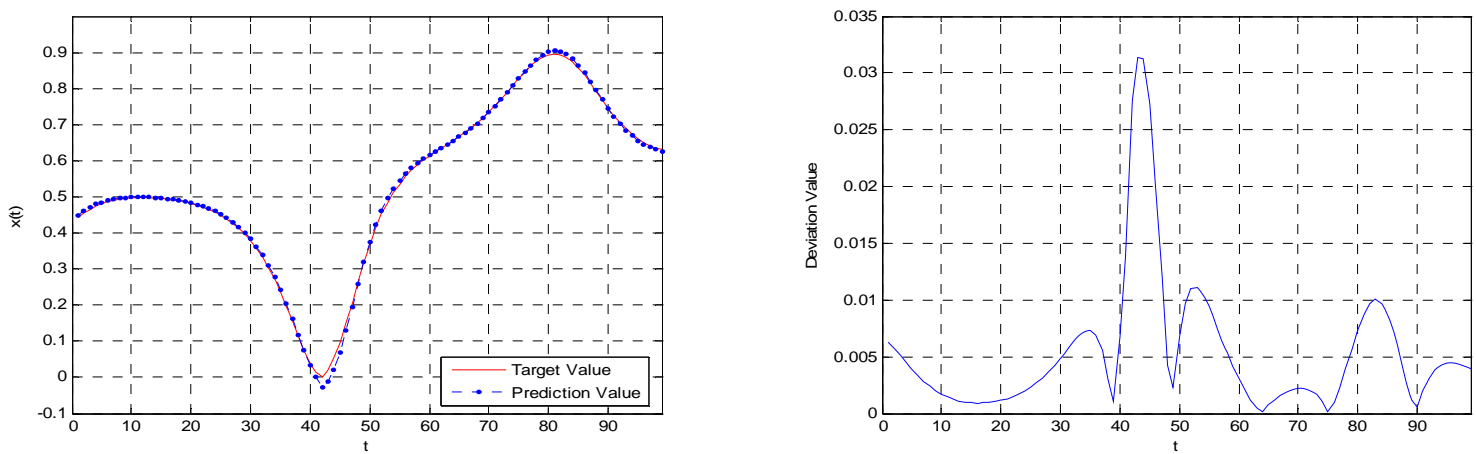

Fig. 16. The prediction value and the deviation value of Lorenz time series.
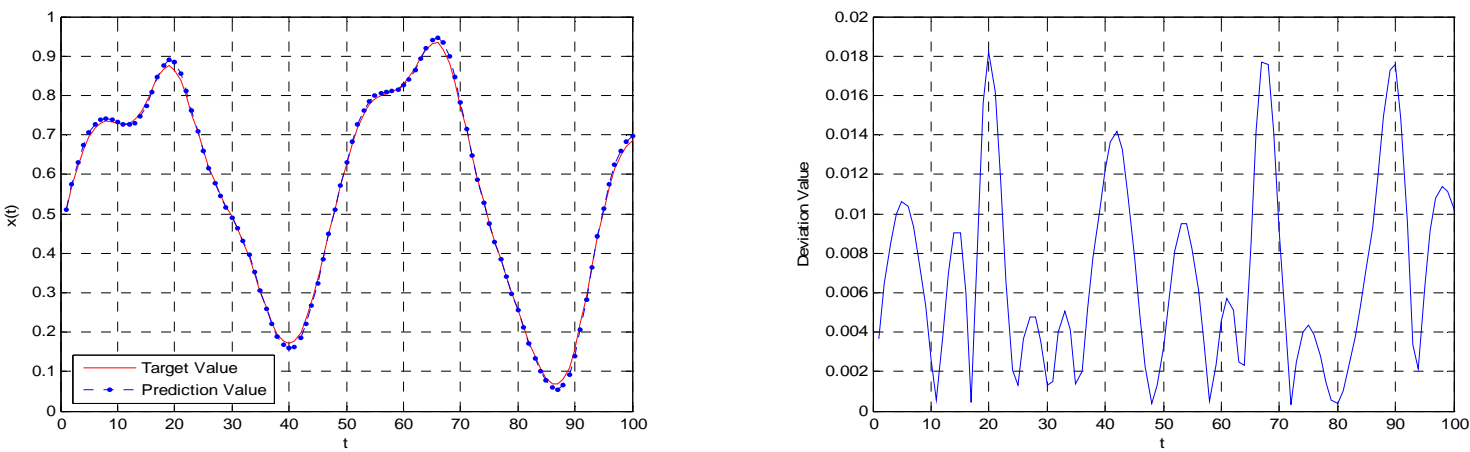

Fig. 17. The prediction value and the deviation value for Mackey-Glass time series.
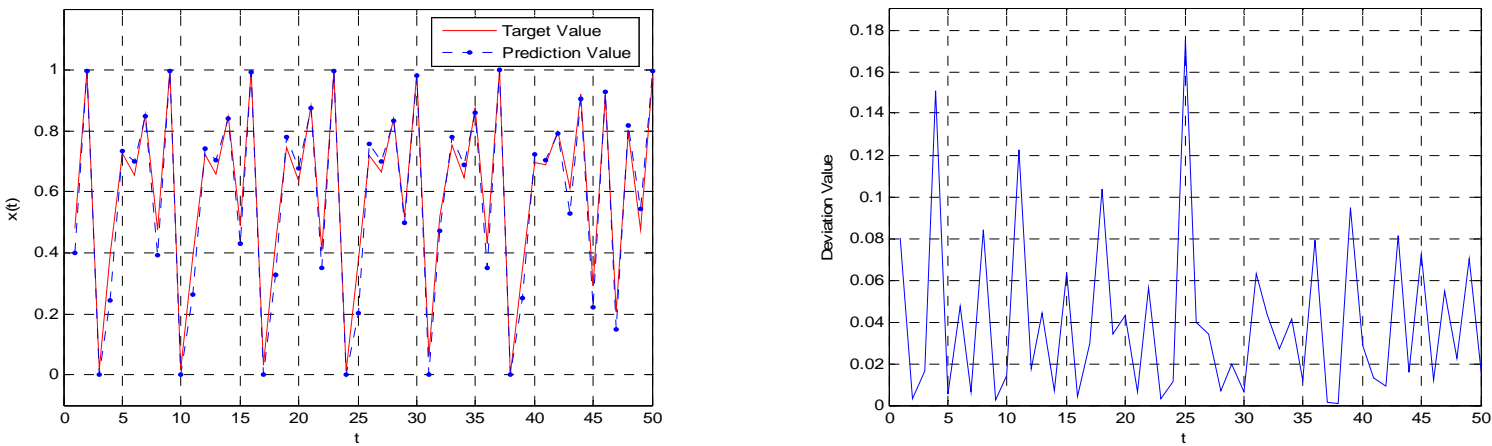

Fig. 18. The prediction value and the deviation value for Henon time series.

reconstruct respectively the phase spaces for corresponding time series, and the sample space scales of three time series are respectively 291,284 and 140 . In the process of time series experiments, we select respectively 100,100 and 50 last sample points from three sample spaces as a prediction sample subset to demonstrate the effectiveness of PIOT, and the rest of sample spaces are considered as a training sample subset, namely selecting a part of training samples being close to a prediction point to build time series prediction model according to sample window $w$ given by Table 1 . Fig. 16, Fig. 17 and Fig. 18 show respectively the prediction values and the corresponding prediction deviation values of three time series in the case of the single-step prediction, where the prediction deviation value is calculated by Eq. (26). Table 3 show the 
prediction error comparison for the PIOT and the genetic algorithm based prediction approach (namely, PGA) provided by Ref. 6, and meanwhile list the corresponding prediction errors of two prediction approaches when three time series are forecasted in terms of single-step prediction, 20 steps prediction and 50 steps prediction, respectively.

The prediction value and the deviation value of Lorenz time series are shown in Fig. 16. The experiment results show that PIOT holds excellent prediction precision for Lorenz time series, in addition, the maximum deviation between target value and prediction value is not exceed 0.0313. At same time, Table 3 lists the single-step prediction results of Lorenz time series, compared with PGA, the prediction precision of PIOT raise one order of magnitude, and its prediction errors, namely RMSE and MAE, are respectively 0.0081 and 0.0053 . For the multi-step prediction of Lorenz time series, we consider two prediction strategies: 20 steps prediction and 50 steps prediction, the experiment results listed in Table 3 show that the prediction errors of PIOT are lower than that of PGA; and the maximum deviations between target value and prediction value of PIOT for two strategies are respectively 0.0472 and 0.0544 .

Fig. 17 depicts the single-step prediction value and the corresponding deviation value of Mackey-Glass time series. Known from the prediction deviation curve in Fig. 17, the deviation value is affected easily by the variation amplitude of Mackey-Glass time series. Compared with the deviation curve of Lorenz time series, the variation amplitude of deviation value for Mackey-Glass time series is big relatively, and its prediction precision is slightly lower than Lorenz time series. However, known from the prediction error given by Table 3 , the both prediction errors have same order of magnitude $10^{-3}$ when two time series are predicted by PIOT according to single-step prediction method; and the variation range for both prediction errors can keep the order of magnitude from $10^{-2}$ to $10^{-3}$ when two time series are predicted by PIOT in terms of 20 steps prediction and 50 steps prediction respectively, so the prediction errors show that PIOT has a good forecasting performance for multi-step prediction. In general, the prediction effectiveness of PIOT is better than that of PGA.

Known from Fig. 7, Henon time series has higher chaotic behavior than the two above-mentioned time series, so its variation rule is more coverable. Fig. 18 depicts the single-step prediction value and corresponding deviation value of Henon time series, the prediction precision of Henon time series is lower relatively than that of Lorenz time series and MackeyGlass time series. But its prediction results can accurately reflect the variation situation of Henon time series in terms of Fig. 18, but simultaneously its prediction errors can keep same order of magnitude with the two above-mentioned time series. In the case of the multi-step prediction, the prediction precision of Henon time series is evidently lower than that of the Lorenz time series and Mackey-Glass time series. Therefore, when using the single-step or multi-step prediction strategies, the prediction effectiveness of PIOT is superior to that of PGA according to the prediction errors given in Table 3 .

From the above-mentioned simulation results, PIOT has better the modelling and predicting capability for Lorenz time series, Mackey-Glass time series and Henon time series. Compared with PGA, PIOT holds good prediction effectiveness for not only single-step prediction but multi-step prediction.

\section{Conclusion}

The combination of chaotic time series prediction principle and immune optimization learning method, this paper propose PIOT prediction approach being used for solving time series prediction problem. In order to obtaining a reasonable prediction model, PIOT use phase space reconstruction method to analyze time series, and use immune optimal method to learn a part of history time series being close to prediction point. In the process of simulation experiments, we select three typical time series, which are Lorenz time series, Mackey-Glass time series and Henon time series, as experimental simulation objectives, and use the singlestep and multi-step prediction method to predict corresponding time series, respectively. The experimental results show that PIOT holds better prediction effectiveness for chaotic time series prediction problem. For single-step prediction situation, compared with PGA, the prediction errors of PIOT decrease one order of magnitude. In addition, the prediction precision of PIOT is easily affected by the time variation of time series in the case of multi-step prediction situation; with the prediction steps increasing, its prediction precision gradually decreasing; but the prediction errors of PIOT are evidently lower than that of PGA under the same conditions.

\section{Acknowledgements}

This work was supported by the National Natural Science Foundation of China under Grant No. 60873246; the National Research Foundation for Doctoral Program 
of Higher Education of China under Grant No. 20070610032; and the Cultivation Fund of the Key Scientific and Technical Innovation Project, Ministry of Education of China under Grant No. 708075.

\section{References}

1. P. Bermolen and D. Rossi, Support vector regression for link load prediction, Computer Networks, 53 (2009) 191-201.

2. C. M. Lee and C. N. Ko, Time series prediction using RBF neural networks with a nonlinear time-varying evolution PSO algorithm, Neurocomputing, 73 (2009) 449-460.

3. K. Xu, J. W. Xu and X. J. Ban, Forecasting of some nonstationary time series based on wavelet decomposition, Acta Electronica Sinica, 29(4) (2001) 566-568.

4. A. B. Geva, ScaleNet-multiscale neural-network architecture for time series prediction, IEEE Transactions on Neural Networks, 9(5) (1998) 1471-1482.

5. Y. H. Chen, B. Yang and J. W. Dong, Time-series prediction using a local linear wavelet neural network, Neurocomputing, 69 (2006) 449-465.

6. G. G. Szpiro, Forecasting chaotic time series with genetic algorithms, The American Physical Society, (1997) 25572568.

7. K. D. Oliveira, A Vannucci and E. C. da Silva, Using artificial neural networks to forecast chaotic time series, Physica A, 284 (2000) 393-404.

8. U. Thissen, R. van Brakela, A.P. de Weijer, W.J. Melssen and L. M. C. Buydens, Using support vector machines for time series prediction, Chemometrics and Intelligent Laboratory Systems, 69 (2003) 35-49.

9. B. Liu and D. P. Hu, Studies on applying artificial neural networks to some forecasting problems, Jounal of Systems Engineering, 14(4) (1999) 338-344.

10. S.S. Keerthi, Efficient tuning of SVM hyper parameters using radius/margin bound and iterative algorithms, IEEE Transactions on Neural Networks, 13(5) (2002) 1225-229.

11. I. Aydin, M. Karakose and E. Akin, A multi-objective artificial immune algorithm for parameter optimization in support vector machine, Applied Soft Computing, 11(1) (2011) 120-129.

12. T. W. Quan, X. M Liu and Q. Liu, Weighted least squares support vector machine local region method for nonlinear time series prediction, Applied Soft Computing, 10 (2010) 562-566.

13. L. N. De Castro and J. Timmis, Artificial immune systems: A new computational intelligence approach (SpringerVerlag, London, 2002).

14. T. Li, Computer Immunology (House of Electronics Industry, Beijing, 2004).
15. J. Timmis, A. Hone, T. Stibor and E. Clark, Theoretical advances in artificial immune systems, Theoretical Computer Science, 403 (2008) 11-32.

16. T. Li, An immunity based network security risk estimation, Science in China Ser F Information Sciences, 48(5) (2005) 557-578.

17. S. Forrest, A. S. Perelson, L. Allen and R. Cherukuri, Self-nonself discrimination in a computer, Proceedings of IEEE Computer Society Symposium on Research in Security and Privacy (IEEE, USA, 1994), pp. 202-212, 1994.

18. L. N. De Castro and F. J. Von Zuben, The clonal selection algorithm with engineering applications, In Workshop Proceedings of GECCO'00, Workshop on Artificial Immune Systems and Their Applications (Las Vegas, USA, 2000), pp. 36-37.

19. X. Shen, X. Z. Gao and R. F. Bie, Artificial immune networks: models and applications, International Journal of Computational Intelligence Systems, 1(2) (2008) 168176.

20. X. Z. Gao, S. J. Ovaska and X. Wang, Re-editing and censoring of detectors in negative selection algorithm, International Journal of Computational Intelligence Systems, 2(3) (2009) 298-311.

21. M. G. Gong, L. C. Jiao, L. N. Zhang and H. F. Du, Immune secondary response and clonal selection inspired optimizers, Progress in Natural Science, 19 (2009) 237 253.

22. Z. H Gan, T. W.S. Chow and W.N. Chau, Clone selection programming and its application to symbolic regression, Expert Systems with Applications, 36 (2009) 3996-4005.

23. N. Sun, Artificial immune optimization algorithm and applications (Harbin Institute of Technology, 2006).

24. N. Khilwani, A. Prakash, R. Shankar and M. K. Tiwari, Fast clonal algorithm, Engineering Applications of Artificial Intelligence, 21 (2008) 106-128.

25. N. H. Packard, J. P. Crutchfietd, J. D. Farmer and R. S. Shaw, Geometry from a time series, Phys. Rev. Lett, 45(9) (1980) 712-716.

26. F. Takens, Detecting strange attractors in turbulence, Lecture notes in Mathematics, 898 (1981) 361-381.

27. H. S. Kim1, R. Eykholt and J. D. Salas, Nonlinear dynamics, delay times, and embedding windows, Physica D, 127 (1999) 48-60.

28. S. Q. Yu, H. H. Wang, N. S. Zhu and R. Ye, Introduction to Immunology (Higher Education Press, Beijing, 2008).

29. A. A. Puntambekar, Data structures and algorithms (Technical Publications Pune, India, 2008).

30. A.J.T. George and D. Grey, Receptor editing during affinity maturation, Immunology Today, 20 (4) (1999) 196.

31. G. Rudolph, Convergence analysis of canonical genetic algorithms, IEEE Trans. Neural Networks, 5 (1994) 96101. 